\title{
Generation of EPR pairs and interconversion of static and flying electron spin qubits
}

\author{
G. Giavaras, ${ }^{1,2, *}$ J. H. Jefferson, ${ }^{2}$ M. Fearn, ${ }^{2}$ and C. J. Lambert ${ }^{1}$ \\ ${ }^{1}$ Department of Physics, Lancaster University, Lancaster LA14YB, England \\ ${ }^{2}$ QinetiQ, St.Andrews Road, Malvern WR143PS, England
}

(Dated: May 11, 2018)

\begin{abstract}
We propose a method of generating fully entangled electron spin pairs using an open static quantum dot and a moving quantum dot, realised by the propagation of a surface acoustic wave (SAW) along a quasi-one-dimensional channel in a semiconductor heterostructure. In particular, we consider a static dot (SD) loaded with two interacting electrons in a singlet state and demonstrate a mechanism which enables the moving SAW-dot to capture and carry along one of the electrons, hence yielding a fully entangled static-flying pair. We also show how with the same mechanism we can load the SD with one or two electrons which are initially carried by a SAW-induced dot. The feasibility of realizing these ideas with existing semiconductor technology is demonstrated and extended to yield flying or static pairs that are fully entangled and arbitrary interconversion of static and flying electron spin qubits.
\end{abstract}

PACS numbers:

Einstein-Podolsky-Rosen (EPR) particle pairs are spatially separated and fully entangled particles such as photons or electrons 1 . A spin entangler system, which is a system capable of generating EPR spin pairs, is of fundamental importance not only in the field of quantum computation but also in quantum mechanics for testing

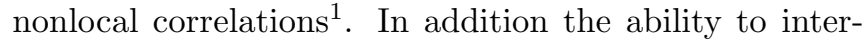
convert static and flying qubits is a basic requirement for quantum computation and communication in general 2 . In the solid state both the creation of an entangler for massive particles and interconversion of qubits are hard tasks since the process of generation and detection must take place in a controlled way and in a much shorter time than typical decoherence times. Electron spin qubits in semiconductors are particularly promising since their decoherence times can be quite long, e.g. $\sim 100 \mathrm{~ns}$ in $\mathrm{GaAs}^{3}$, and a number of entangler schemes have been studied, at least theoretically. These for example include EPR pairs via Coulomb scattering ${ }^{4}$, a triple quantum dot structure $\mathrm{e}^{5,6}$, a three-port $\operatorname{dot}^{\underline{7}}$, a superconductor attached to a double $\operatorname{dot}^{-8}$ and a turnstile double dot device $\frac{9,10,11,12}{}$.

As has been demonstrated experimentally the propagation of a surface acoustic wave (SAW) along a depleted one-dimensional channel in a GaAs/AlGaAs twodimensional electron gas (2DEG) results in the formation of moving quantum dots 13,14 . The heterostructure confines the electrons in the plane of the quantum well, whereas lateral confinement in one direction is due to the channel and in the other direction to the induced time dependent electrostatic potential which accompanies the SAW mechanical propagation. The moving SAW-induced dots can capture electrons from the 2DEG and transport them through the channel. Under a regime of parameters of SAW power and gate voltage the number of transported electrons by the SAW can be controlled down to one yielding a quantized acoustoelectric current of $\mathrm{nA}$ with an accuracy of five parts in $10^{4} \underline{13,14,15}$ In general, this current can be expressed as nef, where $n=1,2 \ldots$, is the average number of electrons in the SAW- dots, $f \sim 2.7 \mathrm{GHz}$ is the SAW frequency and $e$ is the absolute electronic charge. Spin qubits in SAW-dots have been investigated as a possible realisation of a quantum computer $16,17,18$ and recently it was demonstrated theoretically that maximal entanglement can arise from the interaction between a SAW spin qubit and a spin qubit trapped in a static dot (SD), embedded in the depleted channel along which the SAW propagates 19 . Experimentally the interaction between SAW-dots and a SD has been examined mainly in terms of the acoustoelectric current ${ }^{20.21 .22}$ though the main goal is the fabrication of a SAW-based device for quantum information processing 23 .

In this work we demonstrate the feasibility of generating fully entangled pairs of electron-spin qubits to very high fidelity. This differs fundamentally from our earlier work $^{19}$ for which the degree of entanglement was highly dependent on initial conditions, with associated uncertainty in a realistic device. In addition, we demonstrate an efficient mechanism for interconverting the resulting spin qubits from static to flying and vice-versa, an issue which has not been addressed in solid state systems. The basic idea underlying the present proposal is to use a SAW in conjunction with a synchronised variation in gate potential to either eject or capture electrons in a SD in a controlled way. This may be used to generate EPR pairs by first loading the SD with two electrons which are allowed to relax to their singlet ground state. As shown schematically in Fig. 1, one electron is then ejected into a SAW-dot resulting in a delocalized static-flying pair of fully entangled spin-qubits. By the same method, the flying qubit may be subsequently captured by another SD or the static qubit may be ejected into another SAWdot and in this way arbitrary interconversion of static and flying spin qubits may be achieved. The SAW-based loading mechanism of the SD, which is also efficient for two electrons, may be employed in static-flying spin qubit interactions $19,24,25$

The two-electron problem is studied within the effective mass approximation by considering the parabolic 
(a)

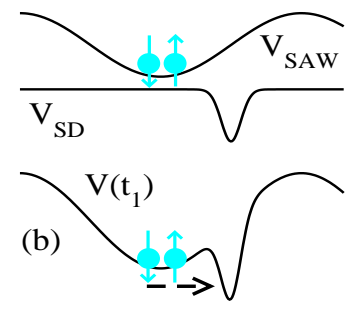

(d)

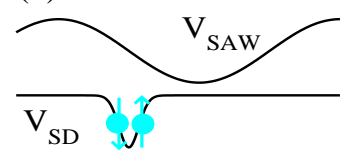

(e)
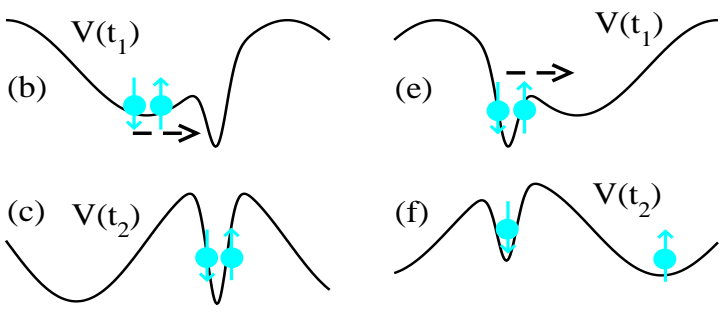

FIG. 1: (color on line). Schematic illustration of the proposed system to generate EPR electron spin pairs. (a) An empty static dot (SD) with a confining potential, $V_{S D}$, is formed in a depleted GaAs/AlGaAs one-dimensional channel. A surface acoustic wave (SAW) induces a sine-wave potential, $V_{S A W}$, which propagates along the channel (from left to right) forming moving quantum dots. A SAW-dot is adjusted to contain two electrons which in general may not be in a singlet state. (b) The total potential $V=V_{S A W}+V_{S D}$ enables tunneling whose degree can be efficiently controlled using a time dependent gate voltage which tunes the depth of the SD. (c) Within a regime of parameters the two electrons can tunnel from the SAW-dot into the SD and can remain bound as the SAW propagates (alternatively the electrons can tunnel into the SD sequentially). Then the electrons are allowed to relax to their singlet ground state. (d) The SD contains two electrons in a singlet ground state while the SAW-dot is empty. (e) Due to the time dependent gate voltage controlled tunneling can take place from the SD into the SAW-dot. (f) One electron can tunnel from the SD into the SAW-dot and it can be carried along by the SAW leaving the second electron trapped in the SD, hence generating a static-flying EPR pair. The SD-electron can then be ejected into a SAW-dot that follows generating a flying EPR pair, or the SAW-electron can be captured by a nearby SD generating a static EPR pair.

band Hamiltonian

$$
H=\sum_{i=1,2}\left[-\frac{\hbar^{2}}{2 m^{*}} \frac{\partial^{2}}{\partial x_{i}^{2}}+V\left(x_{i}, t\right)\right]+V_{c}\left(x_{1}, x_{2}\right) .
$$

where $m^{*}=0.067 m_{o}$ is the effective mass of the electrons in GaAs. The total potential is given by the sum of the SAW and the SD time-dependent potentials i.e., $V(x, t)=V_{S A W}(x, t)+V_{S D}(x, t)$. The SAW potential can be modelled efficiently by the form ${ }^{19} V_{S A W}(x, t)=$ $V_{o} \cos [2 \pi(x / \lambda-f t)]$, with the SAW frequency $f=2.7$ $\mathrm{GHz}$ and the SAW wavelength $\lambda=1 \mu \mathrm{m}$. These are typical values for SAW-based single electron transport. Finally, $V_{o}$ is the SAW potential amplitude, which can be controlled experimentally by the applied power to the transducer which is used to generate the $\mathrm{SAW}^{\underline{13}, 14}$. The confining potential of the SD is modelled by the form $V_{S D}(x, t)=-V_{w}(t) \exp \left(-x^{2} / 2 l_{w}^{2}\right)$, where $l_{w}$ determines the width of the dot and $V_{w}(t)$ is the corresponding depth of the dot which is controlled by a time dependent gate

voltage. Below we describe in detail the time dependence of $V_{w}(t)$. We note that the viability of the mechanism described below is independent of the particular form of $V_{S D}(x, t)$. In addition, the Gaussian dot that we have chosen can approximate a parabolic dot well, which is consistent with experiments on gated quantum dots ${ }^{26}$. The Coulomb term is $V_{c}\left(x_{1}, x_{2}\right)=e^{2} / 4 \pi \epsilon_{r} \epsilon_{o} r$ with $r=\sqrt{\left(x_{1}-x_{2}\right)^{2}+\gamma_{c}^{2}}$ and $\gamma_{c}=20 \mathrm{~nm}$, ensuring that excited-state modes in $y$ and $z$ directions have negligible occupation. For GaAs we have taken $\epsilon_{r}=13$.

To determine the two-electron state of the SD when $V_{S A W}=0$, and with a time independent SD potential we diagonalise numerically Hamiltonian (1). The ground state $\Phi\left(x_{1}, x_{2}\right)$ is a singlet, as has been verified experimentally for parabolic quantum dots 26 . This fully spin entangled state has the orbital distribution $\rho=$ $\int\left|\Phi\left(x, x^{\prime}\right)\right|^{2} d x^{\prime}$ shown in the inset of Fig. 3(a) for the parameters $V_{w}=13 \mathrm{meV}, l_{w}=25 \mathrm{~nm}$. The two electrons are close to the weak correlation regime ${ }^{27}$ (the kinetic energy dominates over the Coulomb energy) having an orbital distribution which peaks in the centre of the SD similarly to the non-interacting limit and, more importantly, the ground state singlet is well separated from excited states.

The time evolution of $\Phi\left(x_{1}, x_{2}\right)$ when $V_{S A W}$ is switched on, while keeping $V_{S D}$ fixed in time, is determined by integrating numerically the time-dependent Schrödinger equation. For $V_{o}=5 \mathrm{meV}$ and, for example, during one SAW period, $T=1 / f=0.37 \mathrm{~ns}$, the state remains well-localized in the SD without tunneling and the electron distribution within the SD is hardly changed. In particular, the state evolves via non-adiabatic LandauZener transitions (LZTs) which enable the state to retain its initial character by changing in time the instantaneous eigenstate index $(n \rightarrow n \pm 1)$ at each anticrossing point 28,29 . To quantify this behaviour we have determined the instantaneous eigenspectrum $E_{n}=$ $E_{n}(t), n=0,1 \ldots$ by solving the eigenvalue problem $H(t) \Phi_{n}(t)=E_{n}(t) \Phi_{n}(t)$ (for singlet states) at each instant in time treating $t$ as parameter. The LZT probability at an anticrossing point with energy gap $2 \delta$ is given by an approximate expression $28.29 P_{L Z} \sim 1-2 \pi \delta^{2} / \hbar \Lambda$, where the matrix element $\Lambda=<\Phi_{e}|\partial H / \partial t| \Phi_{l}>$ involves the states $\Phi_{e}, \Phi_{l}$ which enter and leave the anticrossing point respectively and for the problem under study typical values are $P_{L Z}>0.99$. The number of anticrossing points for a fixed $V_{o}$ increases with decreasing $V_{w}$ and $P_{L Z}$ decrease since the tunnel barrier between SD and SAW-dot weakens $\frac{19}{}$. In the limit of a very small $V_{w}$ the initial electron state cannot follow the SAW propagation even for one cycle, since due to the strong tunneling, it spreads into the continuum immediately when the SAW interacts with the SD. In the opposite limit of a very large $V_{w}$ the two-electron state $\Phi\left(x_{1}, x_{2}\right)$ evolves adiabatically and specifically it corresponds to the ground state at all times and therefore the evolution involves no anticrossing points.

The spin entangler mechanism that we propose using 

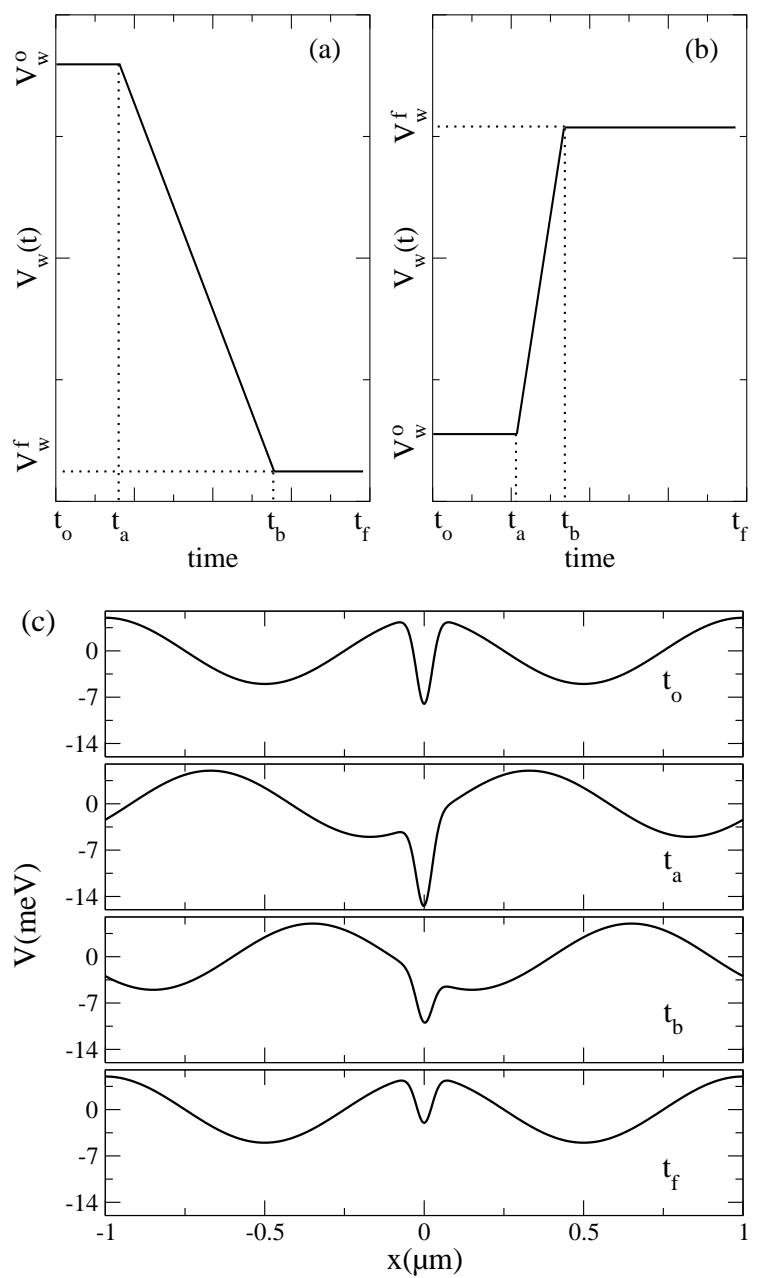

FIG. 2: (a) Variation of the SD depth, which is due to the time dependent gate voltage, when electrons are transferred from the SD to the SAW-dot. (b) The same as in (a) but when electrons are transferred from the SAW-dot to the SD. In both cases $V_{w}^{o}$ is the initial depth of the $\mathrm{SD}, V_{w}^{f}$ is the final depth of the SD and the tuning takes place from $t_{a}$ to $t_{b}$. These quantities are not in general the same for both processes. (c) Total potential for four different times when electrons are transferred from the SD to the SAW-dot. Note that in this case the electrons are bound at $t_{o}=0$ in the SD $(x=0)$ whose depth decreases in time from $t_{a}$ to $t_{b}$. On the other hand, when electrons are transferred from the SAW-dot to the SD they are bound at $t_{o}=0$ in the SAW-dot $(x=-0.5$ $\mu \mathrm{m})$ and the SD potential increases in time from $t_{a}$ to $t_{b}$. The SAW propagates always from left to right.

Hamiltonian (1), which conserves the symmetry of the state in time, is feasible as long as the resulting total time-dependent potential changes in such a manner so as to allow one electron to tunnel from the SD into the SAW-dot during a period of time for which the LZTs occur with negligible probability. On the other hand, after that period of time the potential must enable LZTs with high probability in order that the electron remaining in the SD remains well-localised as the SAW propagates.
We have verified that such behaviour can be achieved by investigating the problem numerically for a typical range of parameters. A simple technique to tune the total potential in this way is to decrease the depth of the $\mathrm{SD}$ with a linear gate voltage ramp. In this work we have used

$$
V_{w}(t)=\left\{\begin{array}{cl}
V_{w}^{o}, & t \leq t_{a} \\
V_{w}^{o}-\Delta_{w} \frac{\left(t-t_{a}\right)}{t_{b}-t_{a}}, & t_{a} \leq t \leq t_{b} \\
V_{w}^{o}-\Delta_{w}, & t \geq t_{b}
\end{array}\right.
$$

where $\Delta_{w}=V_{w}^{o}-V_{w}^{f}$. In this expression $V_{w}^{o}$ is the initial depth of the SD, $V_{w}^{f}$ is the final depth of the SD and the tuning takes place from $t_{a}$ to $t_{b}$. In Fig. 2(a) we show how the variation of the SD depth, $V_{w}(t)$, takes place in time when electrons are transferred from the SD to the SAWdot. For comparison we also show in Fig. 2(b) $V_{w}(t)$ for the inverse process i.e., when electrons are transferred from the SAW-dot to the SD a process that we demonstrate below. The important point is that $V_{w}(t)$ decreases when electrons are transferred from the SD to the SAW$\operatorname{dot}\left(\Delta_{w}>0\right)$, whereas $V_{w}(t)$ increases when electrons are transferred form the SAW-dot to the SD $\left(\Delta_{w}<0\right)$. Figure 2(c) illustrates the variation of the total time dependent potential for four different times when electron transfer from the SD to the SAW-dot takes place. In this case the electrons are bound at $t_{o}$ in the SD $(x=0)$ whose depth decreases in time from $t_{a}$ to $t_{b}$. When electron transfer from the SAW-dot to the SD takes place the electrons are bound at $t_{o}=0$ in the SAW-dot $(x=-0.5$ $\mu \mathrm{m})$ and the SD depth increases in time from $t_{a}$ to $t_{b}$. Note that in both cases the SAW propagates from left to right.

First we describe the EPR pair generation where at the initial time $t_{o}=0$ the two electrons are bound in the SD confining potential. In Fig. 3(a) we show the relevant occupation probabilities at the final time $t_{f}=T=0.37$ ns for different final SD depths, $V_{w}^{f}$, but with an initial depth $V_{w}^{o}=13 \mathrm{meV}$ for all cases. In this example $t_{a}=0.45 T=0.17 \mathrm{~ns}$ and $t_{b}=0.65 T=0.24 \mathrm{~ns}$. A key observation for the efficacy of the entangler is that the SD potential, $V_{S D}$, must change as the potential minimum of the SAW-dot sweeps past it, otherwise the fraction of the initial state which tunnels off the SD cannot be trapped and carried along by the SAW-dot. This requires that $T_{p}=t_{b}-t_{a}$ is of order $T$, which is within the limit of available electronics. Even though for all $V_{w}^{f}$ in Fig. 3 the SD can bind two electrons when $V_{S A W}=0$, the physical effect of the time dependent gate voltage when combined with the SAW potential is to decrease in time the binding energy of the SD forcing one electron to tunnel partly or even totally into the SAW-dot. This is why $P_{S D, S D}$ decreases with decreasing $V_{w}^{f}$ whilst $P_{S D, S A W}$ increases. In this study the range that we vary $V_{w}^{f}$ ensures that a single electron can successfully accomplish LZTs, following the SAW propagation without tunneling, therefore $P_{S A W, S A W} \sim 0$. On the other hand, when dou- 

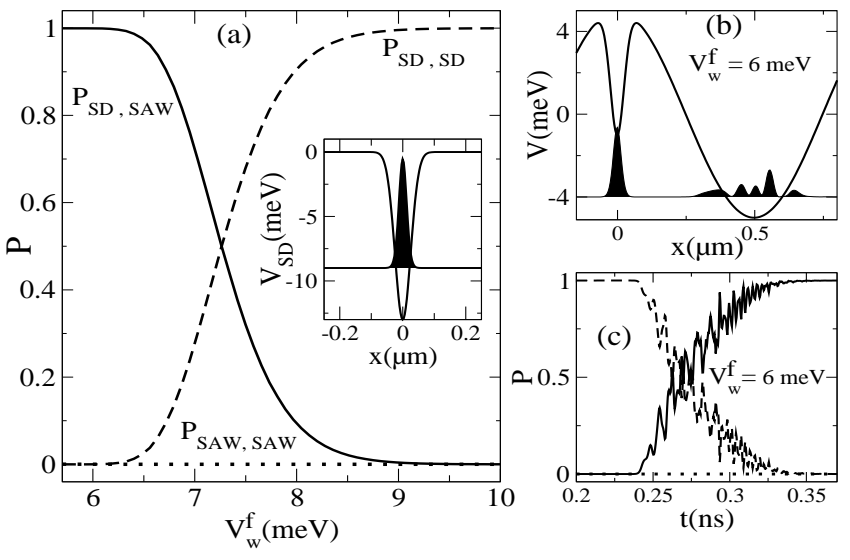

FIG. 3: (a) Occupation probabilities at the final time $t_{f}=$ $T=0.37 \mathrm{~ns}$ as a function of the final depth of the SD, when the initial depth of the SD is $V_{w}^{o}=13 \mathrm{meV}, t_{a}=0.45 T=0.17$ $\mathrm{ns}, t_{b}=0.65 T=0.24$ and at the initial time $t_{o}=0$ the two electrons are trapped in the SD. $P_{S D, S D}$ is the probability of finding the two electrons in the SD, $P_{S A W, S A W}$ is the probability of finding the two electrons in the SAW-dot and $P_{S D, S A W}$ is the probability of finding one electron in the SD and the other in the SAW-dot. The inset shows the initial $\left(t_{o}=0\right)$ two-electron distribution (in arbitrary units) in the SD when $V_{S A W}=0$. (b) Total potential and two-electron distribution at final time $t_{f}=T=037 \mathrm{~ns}$, when $V_{w}^{f}=6 \mathrm{meV}$ and at $t_{o}=0$ the two electrons are trapped in the SD. (c) The same as in (a) but as a function of time when $V_{w}^{f}=6 \mathrm{meV}$.

ble occupation in the SD is appreciable, uncontrollable tunneling off the SD can still occur after $t_{f}=T=0.37$ ns. This can be minimized by restoring the SD potential to its original depth with the gate, though this regime with appreciable $P_{S D, S D}$ is not appropriate for the entangler. We can achieve a nearly ideal operation mode for $V_{w}^{f} \lesssim 6 \mathrm{meV}$ which, as shown in Fig. 3(a), at the final time $t_{f}=T=0.37 \mathrm{~ns}$ results in one trapped electron in the SD and a second electron trapped in the moving SAW-dot with very high probability $P_{S D, S A W}>0.99$. However, we point out that when the SD depth is made too shallow $\left(V_{w}^{f} \lesssim 3 \mathrm{meV}\right)$ both electrons tunnel into the SAW-dot and $\widetilde{P}_{S A W, S A W}=1$. For the case $V_{w}^{f}=6 \mathrm{meV}$ Fig. 3(b) shows the final electron distribution when the Coulomb interaction is negligible and Fig. 33(c) shows the occupation probabilities as a function of time ${ }^{30}$. The SD-state is the corresponding lowest eigenstate, whereas the SAWstate consists of a superposition of the lowest SAW-dot eigenstates whose number can be controlled with choice of $T_{p}$, SD and SAW parameters. At the final time the SD-state develops via non-adiabatic LZTs, whereas the SAW-state develops adiabatically, to a good approximation, with no leakage due to the high confinement provided by $V_{o} \underline{19}$.

The electron which remains trapped in the SD can be made to follow the SAW-electron by further decreasing the depth of the SD and releasing the trapped electron in a SAW-dot that follows. In this way we may cre-

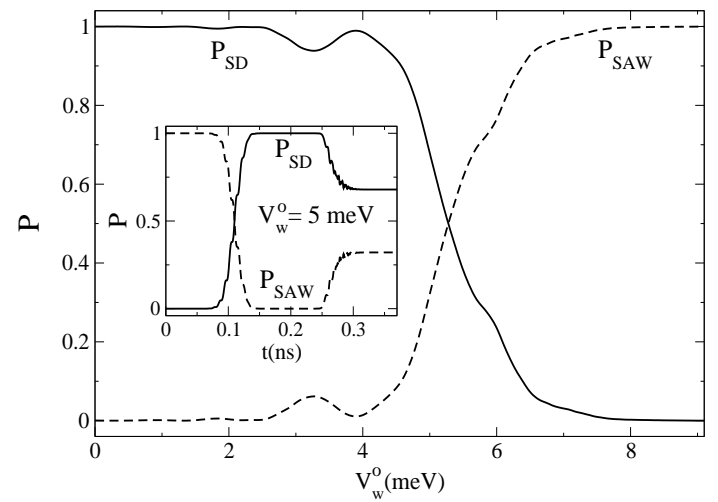

FIG. 4: Occupation probabilities at final time $t_{f}=T=0.37$ ns as a function of the initial depth of the SD, when the final depth of the SD is $V_{w}^{f}=11 \mathrm{meV}, t_{a}=0.39 \mathrm{~T}=0.14$ $\mathrm{ns}, t_{b}=0.58 T=0.21 \mathrm{~ns}$ and at the initial time $t_{o}=0$ one electron is carried by the SAW-dot. $P_{S D}$ is the probability of loading the SD with a single electron and $P_{S A W}$ is the probability of keeping the electron in the SAW-dot. The inset shows a typical example of the time evolution when $V_{w}^{o}=5$ $\mathrm{meV}$.

ate flying entangled nonlocal electron pairs travelling at the sound velocity $\lambda f=2700 \mathrm{~ms}^{-1}$. Since the wave packets are trapped in the SAW-dots, any physical spreading to neighboring SAW-dots is negligible. In fact the overlap between states of neighboring SAW-dots can be controlled and even completely minimised by choosing a very strong SAW potential amplitude. If necessary for measurement the two electrons can be driven to different channels using a Y-branch design ${ }^{31}$ or isolated SAWpulses 16 , to avoid interference effects. Detection and measurement of the flying SAW-dot spins can be performed with the techniques of Refs. 16 and 17. Another option (described below) is to trap the SAW-electron in a nearby SD using a synchronised time-dependent gate voltage yielding a nonlocal spin singlet with one electron each in two spatially delocalized SDs. Measurement of the SD-spins can then be made using the spin to charge conversion method 32 and finally the spins can be removed via tunneling or by a SAW to allow repetition of the cycle. In either case the empty SD may be loaded with two electrons from leads or by using a SAW, as we show below, and the whole process repeated.

We now present results on the loading process of a SD with one or two electrons using a moving SAW-dot. The former process enables spin conversion from flying to static, while the latter can be the starting point for EPR pair generation described above. For this purpose a SAW-dot carrying at $t_{o}=0$ one or two electrons ${ }^{33}$ is driven towards the empty SD whose confining potential is increased synchronously with a time dependent gate voltage according to Eq.(2). Specifically, in Fig. 4 we show the relevant occupation probabilities at the final time $t_{f}=T=0.37 \mathrm{~ns}$ (similar behaviour is displayed for $t>t_{f}$ ) as a function of $V_{w}^{o}$ for a single electron which at 


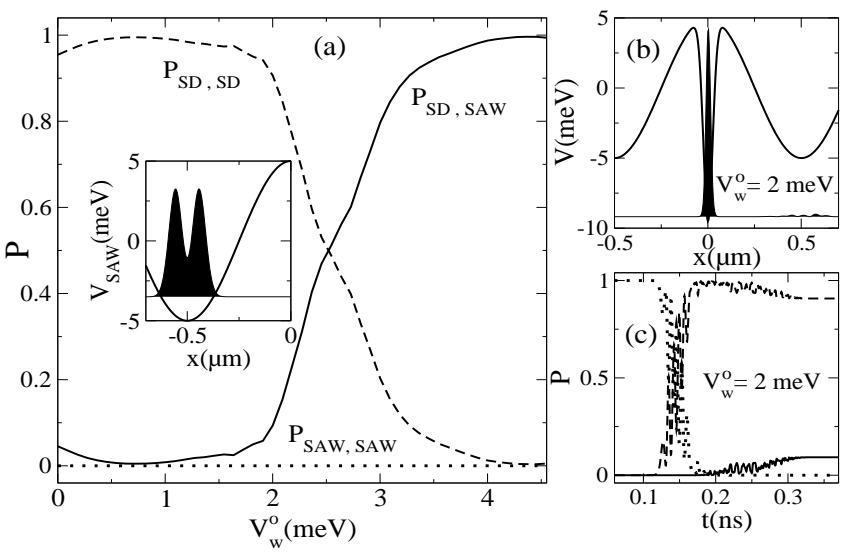

FIG. 5: (a) Occupation probabilities at the final time $t_{f}=$ $T=0.37 \mathrm{~ns}$ as a function of the initial depth of the SD when the final depth of the SD is $V_{w}^{f}=14.5 \mathrm{meV}, t_{a}=0.42 T=0.16$ $\mathrm{ns}, t_{b}=0.7 T=0.26 \mathrm{~ns}$ and at the initial time $t_{o}=0$ the two electrons are carried by the SAW-dot. $P_{S D, S D}$ is the probability of loading with two electrons the SD, $P_{S A W, S A W}$ is the probability of keeping the two electrons in the SAW-dot and $P_{S D, S A W}$ is the probability of loading with a single electron the SD, while the second electron remains in the SAW-dot. The inset shows the initial $\left(t_{o}=0\right)$ two-electron distribution (in arbitrary units) in the SAW-dot. (b) Total potential and two-electron distribution at final time $t_{f}=T=037 \mathrm{~ns}$, when $V_{w}^{o}=2 \mathrm{meV}$ and at $t_{o}=0$ the two electrons are carried by the SAW-dot. (c) The same as in (a) but as a function of time when $V_{w}^{o}=2 \mathrm{meV}$.

$t_{o}=0$ is in the ground state of the SAW-dot. The inset of Fig. 4 shows the occupation probabilities as a function of time when $V_{w}^{o}=5 \mathrm{meV}$. Note that for the whole range of $V_{w}^{o}$ in Fig. 4 the final depth of the SD is $V_{w}^{f}=11$ $\mathrm{meV}$ and $t_{a}=0.39 T=0.14 \mathrm{~ns}, t_{b}=0.58 T=0.21 \mathrm{~ns}$. The loading mechanism is efficient since it can induce a high loading probability i.e. $P_{S D}>0.99$ for $V_{w}^{o} \lesssim 2 \mathrm{meV}$. By increasing $V_{w}^{o}$ higher eigenstates mediate the tunneling process resulting in smaller $P_{S D}$ since the width of the tunnel barrier between SD and SAW-dot increases and inhibits electron tunneling into SD-states which can follow LZTs. For the higher excited SD states the process is not efficient because the electron tunnels in, and subsequently off, the SD without following LZTs. For a deeper $V_{w}^{f}$ additional excited states can be made to follow LZTs, and thus to enhance the range for which $P_{S D}$ is close to unity. We have also verified the robustness of the loading mechanism for cases in which the initial SAW-dot state is a random superposition of the low-lying SAW-eigenstates ${ }^{34}$. We have further verified that a second SAW-electron (which is in the ground state of the SAW-dot) may be loaded successfully in the SD when the first electron is assumed to be in the SD ground state and $V_{w}(t)$ is increased according to Eq.(2). In principle, this can happen for both singlet and triplet states, however, $V_{w}^{f}$ must be deeper than for the loading of the first electron since the second electron has to overcome the mutual Coulomb repulsion with the first. In this way, the SD may be loaded with two electrons which, after relaxation to the singlet ground state, becomes the starting point for EPR pair generation described earlier.

In Fig. 5 we extend the results to two electrons carried at $t_{o}=0$ in a single SAW-dot in the corresponding singlet ground state, whose orbital distribution $\rho=$ $\int\left|\Psi\left(x, x^{\prime}\right)\right|^{2} d x^{\prime}$ is shown in the inset of Fig [5(a). This may be used, for example, as an alternative to sequential loading of the SD prior to EPR pair generation. Similarly to one electron $V_{w}(t)$ is increased according to Eq.(2) and as we see in Fig 5(a) an increase of $V_{w}^{o}$ lowers the occupation probability $P_{S D, S D}$, while $P_{S A W, S A W} \sim 0$ indicating that for this range of $V_{w}^{o}$ there is always enough time for one electron to tunnel into the SD and follow LZTs. For the case $V_{w}^{o}=2 \mathrm{meV}$ Fig. 5 (b) shows the final electron distribution and Fig. 5(c) shows the occupation probabilities as a function of time. Note that for the whole range of $V_{w}^{o}$ in Fig. [5 the final depth of the SD is $V_{w}^{f}=14.5$ $\mathrm{meV}$ and $t_{a}=0.42 T=0.16 \mathrm{~ns}, t_{b}=0.7 T=0.26 \mathrm{~ns}$. In general, the Coulomb repulsion between the electrons in the SAW-dot enhances the tunneling process into the SD for the first electron, but the second electron has then to overcome the Coulomb repulsion of the first, as with sequential loading described above. If $V_{w}^{o}$ is sufficiently deep, the second electron tunnels in and off the SD [righthand side of Fig. [5(a)] and, as excited states mediate the process, the electron remaining in the SAW-dot is left in a superposition of the lowest SAW-eigenstates. Unlike the narrow SD, the potential minimum of the SAW-dot is relatively wide and as a result the Coulomb energy dominates over the kinetic energy and hence the two electrons are strongly correlated. For this reason they occupy relatively distinct regions in the SAW-dot, as shown in the inset of Fig. 5(a) and in fact both singlet and triplet states have almost identical distributions yielding a small antiferromagnetic exchange energy. This small energy splitting of singlet and triplet leads to a high uncertainty in the degree of entanglement of the incident electrons in the SAW-dot which in turn leads to a high uncertainty in the resulting static-flying qubit pair. This would not, therefore, be such an effective procedure for producing fully entangled EPR pairs, as the method described earlier which ensures relaxation to the singlet ground state within the SD due to the much higher energy splitting of singlet and triplet. However, we have proved that by tuning the depth of the SD synchronously with the moving SAW-dot containing two electrons, these electrons may both be bound in the SD with high probability, as shown in Fig. 5(a), confirming this as an alternative two-electron loading strategy of a SD.

Finally, we point out that the electron spins are subjected to decoherence as in all solid state systems. The spin lifetime in GaAs is $~ 100$ ns arising primarily from phonon scattering $\underline{3}$. In our proposal the time scales of interest of $\sim 0.4 \mathrm{~ns}$ as seen from Figs. 3(c) and 5(c), are more than two orders of magnitude shorter. Decoherence and error mechanisms for relevant SAW-based 
devices have been described in Refs. 16 and 17 and include for example coupling of electron spins to nuclear spins ${ }^{35}$, noise on surface gates, impurities and disorder, errors in the width of the depleted channel and temperature effects. A detailed study of decoherence effects for our scheme will be presented elsewhere.

In conclusion, we have proposed and studied an electron spin entangler which generates in a controlled way nonlocal singlet states and can be realised with existing semiconductor technology. The entangler scheme utilizes a synchronized time dependent gate voltage which tunes the confining potential of a SD formed in a depleted channel, and a SAW-dot which propagates through the channel and captures (transfers) electrons from (to) the SD. By studying the exact time evolution of two-electron states we showed that static-flying EPR electron spin pairs, which may be converted to static or flying, can be efficiently produced within times of $0.4 \mathrm{~ns}$. We also demonstrated a SAW scheme of loading a SD with one and two electrons, enabling arbitrary interconversion of flying and static spin qubits bound in a quatum dot.

We thank M. Kataoka for discussions. This work was supported by the UK MoD and is part of the UK QIP IRC (www.qipirc.org, GR/S82176/01).
* Present address: Department of Physics and Astronomy, University of Leicester, Leicester, England

1 A. Einstein, B. Podolsky, and N. Rosen, Phys. Rev. 47, 777 (1935).

2 D. P. DiVincenzo, Fortschr. Phys. 48, 771 (2000).

3 J. M. Kikkawa and D. D. Awschalom, Phys. Rev. Lett. 80, 4313 (1998).

4 D. S. Saraga, B. L. Altshuler, D. Loss, and R. M. Westervelt, Phys. Rev. Lett. 92, 246803 (2004).

${ }^{5}$ P. Zhang, Q.-K. Xue, X.-G. Zhao, and X. C. Xie, Phys Rev. A 69, 042307 (2004).

6 D. S. Saraga and D. Loss, Phys. Rev. Lett. 90, 166803 (2003).

7 W. D. OLiver, F. Yamaguchi, and Y. Yamamoto, Phys. Rev. Lett. 88, 037901 (2002).

${ }^{8}$ P. Recher, E. V. Sukhorukov, and D. Loss, Phys. Rev. B 63, 165314 (2001).

9 G. Burkard, D. Loss, and E. V. Sukhorukov, Phys. Rev. B 61, 16303(R) (2000).

10 X. Hu and S. Das Sarma, Phys. Rev. B 69, 115312 (2004).

11 M. Blaauboer and D. P. DiVincenzo, Phys. Rev. Lett. 95, 160402 (2005).

12 S. Legel, J. Konig, G. Burkard, and G. Schon, Phys. Rev. B 76, 085335 (2007).

13 J. M. Shilton, V. I. Talyanskii, M. Pepper, D. A. Ritchie, J. E. F. Frost, C. J. B. Ford, C. G. Smith, C. G. Ford, and G. A. C. Jones, J. Phys. Condens. Matter 8, L531 (1996).

14 V. I. Talyanskii, J. M. Shilton, M. Pepper, C. G. Smith, C. J. B. Ford, E. H. Linfield, D. A. Ritchie, and G. A. C. Jones, Phys. Rev. B 56, 15180 (1997).

15 J. Cunningham, V. I. Talyanskii, J. M. Shilton, M. Pepper, M. Y. Simmons, D. A. Ritchie, Phys. Rev. B 60, 4850 (1999).

16 C. H. W. Barnes, J. M. Shilton, and A. M. Robinson, Phys. Rev. B 62, 8410 (2000).

17 S. Furuta, C. H. W. Barnes, and C. J. L. Doran, Phys. Rev. B 70, 205320 (2004).

18 G. Gumbs and Y. Abranyos, Phys. Rev. A 70, 050302(R) (2004).

19 G. Giavaras, J. H. Jefferson, A. Ramšak, T. P. Spiller, and C. J. Lambert, Phys. Rev. B 74, 195341 (2006).

20 J. Ebbecke, N. E. Fletcher, T. J. B. M. Janssen, H. E. Beere, D. A. Ritchie, and M. Pepper, Phys. Rev. B 72,
$121311(2005)$.

${ }^{21}$ J. Ebbecke, N. E. Fletcher, T. J. B. M. Janssen, F. J. Ahlers, M. Pepper, H. E. Beere, and D. A. Ritchie, Appl. Phys. Lett. 84, 4319 (2004).

${ }^{22}$ N. E. Fletcher, J. Ebbecke, T. J. B. M. Janssen, F. J. Ahlers, M. Pepper, H. E. Beere, and D. A. Ritchie, Phys. Rev. B 68, 245310 (2003).

23 M. Kataoka, R. J. Schneble, A. L. Thorn, C. H. W. Barnes, C. J. B. Ford, D. Anderson, G. A. C. Jones, I. Farrer, D. A. Ritchie, and M. Pepper, Phys. Rev. Lett. 98, 046801 (2007).

24 J. H. Jefferson, A. Ramšak, and T. Rejec, Europhys. Lett. 75, 764 (2006).

25 A. T. Costa, Jr., S. Bose, and Y. Omar, Phys. Rev. Lett. 96, 230501 (2006).

26 S. M. Reimann and M. Manninen, Rev. Mod. Phys. 74, 1283 (2002), and references therein.

27 G. W. Bryant, Phys. Rev. Lett. 59, 1140 (1987).

28 P. A. Maksym, Phys. Rev. B 61, 4727 (2000).

29 C. Zener, Proc. R. Soc. London, Series A 137, 696 (1932).

30 The occupation probabilities shown in this work have meaning only when the SAW-dot and SD regions do not overlap. This is exactly the case at the final time $t_{f}=T=$ $0.37 \mathrm{~ns}$, that we are interested in, when the probabilities have obtained their asymptotic values.

31 V. I. Talyanskii, M. R. Graham, and H. E. Beere, Appl. Phys. Lett. 88, 083501 (2006).

32 R. Hanson, L. H. Willems van Beveren, I. T. Vink, J. M. Elzerman, W. J. M. Naber, F. H. L. Koppens, L. P. Kouwenhoven, and L. M. K. Vandersypen, Phys. Rev. Lett. 94, 196802 (2005).

33 In principle, the number of electrons in the SAW-dots can be controlled accurately with the gate voltage which is applied to form the channel along which the SAW propagates (see for example Refs. 13 and 14).

34 This is useful if we want to convert the static-flying EPR pair to a static pair, because as we saw in Fig. 3(b) the electron which tunnels from the SD to the SAW-dot is in a superposition of SAW-eigenstates.

35 J. R. Petta, A. C. Johnson, J. M. Taylor, E. A. Laird, A. Yacoby, M. D. Lukin, C. M. Marcus, M. P. Hanson, and A. C. Gossard, Science 309, 2180 (2005). 